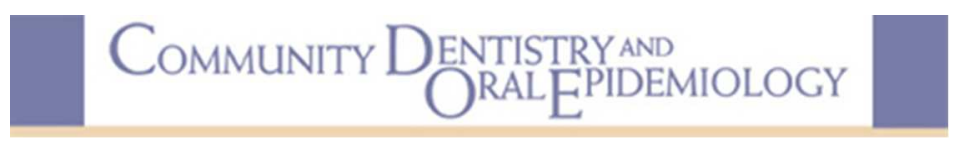

\title{
Experience of childhood abuse and later number of remaining teeth in older Japanese: a life-course study from Japan Gerontological Evaluation Study project
}

\begin{tabular}{|c|c|}
\hline Journal: & Community Dentistry and Oral Epidemiology \\
\hline Manuscript ID & CDOE-16-013.R1 \\
\hline Manuscript Type: & Original Manuscript \\
\hline Date Submitted by the Author: & $\mathrm{n} / \mathrm{a}$ \\
\hline Complete List of Authors: & $\begin{array}{l}\text { Matsuyama, Yusuke; Tohoku University Graduate School of Dentistry, } \\
\text { Department of International and Community Oral Health } \\
\text { Fujiwara, Takeo; National Research Institute for Child Health and } \\
\text { Development, Department of Social Medicine } \\
\text { Aida, Jun; Tohoku University Graduate School of Dentistry, Department of } \\
\text { International and Community Oral Health } \\
\text { Watt, Richard; University College London, Epidemiology and Public Health } \\
\text { Kondo, Naoki; University of Tokyo, School of Public Health, Department of } \\
\text { Health and Social Behavior, Department of Health Education and Health } \\
\text { Sociology } \\
\text { Yamamoto, Tatsuo; Kanagawa Dental University, Graduate School of } \\
\text { Dentistry, Department of Oral Science } \\
\text { Kondo, Katsunori; Chiba University, Center for Preventive Medical } \\
\text { Sciences; Nihon Fukushi University, Center for Well-being and Society } \\
\text { Osaka, Ken; Tohoku University Graduate School of Dentistry, Department } \\
\text { of International and Community Oral Health }\end{array}$ \\
\hline Keywords: & Dental Health, Epidemiology, Tooth loss, Public health \\
\hline Abstract: & $\begin{array}{l}\text { Objectives: From a life-course perspective, adverse childhood experiences } \\
\text { (ACEs) such as childhood abuse are known risk factors for adult diseases } \\
\text { and death throughout life. ACEs could also cause poor dental health in later } \\
\text { life because they could induce poor dental health in childhood, initiate } \\
\text { unhealthy behaviors, and lower immune and physiological functions. } \\
\text { However, it is not known whether ACEs have a longitudinal adverse effect } \\
\text { on dental health in older age. This study aimed to investigate the } \\
\text { association between experience of childhood abuse until the age of } 18 \text { and } \\
\text { current number of remaining teeth among a sample of older Japanese } \\
\text { adults. } \\
\text { Methods: A retrospective cohort study was conducted using the data from } \\
\text { the Japan Gerontological Evaluation Study (JAGES); a large-scale, self- } \\
\text { reported survey in } 2013 \text { including } 27,525 \text { community-dwelling Japanese } \\
\text { aged } \geq 65 \text { years (response rate }=71.1 \%) \text {. The outcome, current number of } \\
\text { remaining teeth was used categorically: } \geq 20,10-19,5-9,1-4, \text { and no } \\
\text { teeth. Childhood abuse was defined as having any experience of physical } \\
\text { abuse, psychological abuse, and psychological neglect up until the age of } \\
18 \text { years. Ordered logistic regression models were applied. } \\
\text { Results: Of the } 25,189 \text { respondents who indicated their number of }\end{array}$ \\
\hline
\end{tabular}




\begin{tabular}{|l|l|}
\hline & remaining teeth (mean age: $73.9 ;$ male: $46.5 \%), 14.8 \%$ had experience of \\
childhood abuse. Distributions of $\geq 20,10-19,5-9,1-4$, and no teeth were: \\
$46.6 \%, 22.0 \%, 11.4 \%, 8.2 \%$, and $11.8 \%$ among respondents with \\
childhood abuse; while $52.3 \%, 21.3 \%, 10.3 \%, 6.6 \%$, and $9.5 \%$ among \\
respondents without childhood abuse. Childhood abuse was significantly \\
associated with fewer remaining teeth after adjusting for covariates \\
including socioeconomic status (odds ratio=1.14; $95 \%$ confidence interval: \\
$\begin{array}{ll}\text { 1.06, } 1.22) . \\
\text { Conclusions: Childhood abuse could have a longitudinal adverse effect on } \\
\text { later dental health in older age. This study emphasizes the importance of } \\
\text { early life experiences on dental health throughout later life. }\end{array}$ \\
\hline
\end{tabular}

SCHOLARONE ${ }^{\text {m }}$

Manuscripts 
Title

Experience of childhood abuse and later number of remaining teeth in older Japanese: a life-course study from Japan Gerontological Evaluation Study project

Authors

Yusuke Matsuyama ${ }^{1)}$, Takeo Fujiwara ${ }^{2)}$, Jun Aida ${ }^{1)}$, Richard G Watt ${ }^{3)}$ Naoki Kondo ${ }^{4)}$, Tatsuo Yamamoto ${ }^{5)}$, Katsunori Kondo ${ }^{6)}$ 7), and Ken Osaka ${ }^{1)}$

Institutional affiliations

1) Department of International and Community Oral Health, Tohoku University Graduate School of Dentistry

2) Department of Social Medicine, National Research Institute for Child Health and Development

3) Department of Epidemiology and Public Health, University College London, London, UK

4) Department of Health and Social Behavior, Department of Health Education and Health Sociology, School of Public Health, University of Tokyo

5) Division of Dental Sociology, Department of Oral Science, Graduate School of Dentistry, Kanagawa Dental University

6) Center for Preventive Medical Sciences, Chiba University

7) Center for Well-being and Society, Nihon Fukushi University, Nagoya, Japan

Running title

Childhood abuse and number of teeth in older Japanese 
Number of tables: 3

Number of figures: 0

Number of photographs: 0

Number of supplemental files: 2

Word count (including abstract and reference) 3,997/4,000

Corresponding author

Yusuke Matsuyama, e-mail: matsuyama-thk@umin.org

Department of International and Community Oral Health, Tohoku University Graduate

School of Dentistry, 4-1, Seiryo-machi, Aoba-ku, Sendai, Miyagi 980-8575, Japan 
$1 \quad$ Abstract

2 Objectives: From a life-course perspective, adverse childhood experiences (ACEs) such

3 as childhood abuse are known risk factors for adult diseases and death throughout life.

4 ACEs could also cause poor dental health in later life because they could induce poor

5 dental health in childhood, initiate unhealthy behaviors, and lower immune and

6 physiological functions. However, it is not known whether ACEs have a longitudinal

7 adverse effect on dental health in older age. This study aimed to investigate the

8 association between experience of childhood abuse until the age of 18 and current

9 number of remaining teeth among a sample of older Japanese adults.

10 Methods: A retrospective cohort study was conducted using the data from the Japan

11 Gerontological Evaluation Study (JAGES); a large-scale, self-reported survey in 2013

12 including 27,525 community-dwelling Japanese aged $\geq 65$ years (response rate $=71.1 \%$ ).

13 The outcome, current number of remaining teeth was used categorically: $\geq 20,10-19,5-$

$149,1-4$, and no teeth. Childhood abuse was defined as having any experience of physical

15 abuse, psychological abuse, and psychological neglect up until the age of 18 years.

16 Ordered logistic regression models were applied.

17 Results: Of the 25,189 respondents who indicated their number of remaining teeth

18 (mean age: 73.9; male: 46.5\%), 14.8\% had experience of childhood abuse. Distributions

19 of $\geq 20,10-19,5-9,1-4$, and no teeth were: $46.6 \%, 22.0 \%, 11.4 \%, 8.2 \%$, and $11.8 \%$

20 among respondents with childhood abuse; while 52.3\%, 21.3\%, 10.3\%, 6.6\%, and 9.5\%

21 among respondents without childhood abuse. Childhood abuse was significantly

22 associated with fewer remaining teeth after adjusting for covariates including

23 socioeconomic status (odds ratio $=1.14 ; 95 \%$ confidence interval: $1.06,1.22$ ). 
24 Conclusions: Childhood abuse could have a longitudinal adverse effect on later dental

25 health in older age. This study emphasizes the importance of early life experiences on 26 dental health throughout later life.

27

28 
Introduction

The World Health Organization has highlighted that a good start to life should

be ensured for every child in order to provide the foundations for health and well-being

and to tackle health inequalities, which can be observed even in the earliest stage of life ${ }^{1}$.

Every child has the right to live a healthy life free from violence ${ }^{2}$. However, many

children have adverse childhood experiences (ACEs). For example, in Japan, 31.9\% of

adults $^{3}$ and worldwide $38.8 \%$ of adults ${ }^{4}$ have experienced one or more ACEs, such as

interpersonal loss, parental substance abuse, physical or sexual abuse, neglect, and

economic adversity during their childhood. Reducing ACEs is therefore an important public health issue ${ }^{1}$.

Life-course epidemiology has highlighted that ACEs are risk factors for later adult diseases and death ${ }^{5}$. Two conceptual life-course models, critical period model and accumulation of risk model, are suggested as possible pathways. In the critical period model, exposures during critical or sensitive periods irreversibly change anatomical structures, such as the hypothalamic-pituitary-adrenal axis, or psychological functions and could result in disease later in life. In the accumulation of risk model, exposures in early life stages and other risk factors accumulate throughout life and, in turn, damage health later in life either independently or by interacting with each other ${ }^{6-8}$.

ACEs could have a longitudinal adverse effect on oral health. In the critical period model, physically abused children's teeth are sometimes injured. Fractured and avulsed teeth were found among physically abused children, suggesting that the teeth of abused children were injured by physical violence from a parent ${ }^{9,10}$. If permanent teeth

51 were injured, later oral health would be influenced. Severe chronic stress induced by

52 ACEs could alter children's hypothalamic-pituitary-adrenal axis function and could 
irreversibly damage their immune systems ${ }^{11}$. A compromised immune system is associated with periodontal disease in adulthood ${ }^{12}$. Individuals who have experienced ACEs tend to exhibit addictive and unhealthy behaviors ${ }^{13}$ because their inhibitory control system has been damaged ${ }^{14}$. Such people tend to consume 'comfort' foods (such as energy-dense foods high in sugar and/or fat) to cope with stress ${ }^{15-17}$. Consumption of these sugary food items is associated with both dental caries and overweight/obesity ${ }^{18}$. These eating habits in childhood could persist into adulthood ${ }^{19,20}$ and might contribute to later poor dental health. Moreover, people with ACEs feel daily stress, which might result in a lower salivary flow rate through sympathetic nerve functioning ${ }^{21}$. A low salivary flow rate is a recognized risk factor for dental caries ${ }^{22}$. Thus, it is reasonable to assume that ACEs might induce poor dental health in adulthood.

Among ACEs, childhood abuse warrants particular attention for dental health later in life since it could cause poor dental health in childhood. Valencia-Rojas et al. (2008) reported an association between child abuse and early childhood caries among Canadian children ${ }^{23}$. Other studies have found an association between high levels of paternal punishment in childhood and dental injuries as well as dental caries experience ${ }^{24,25}$. However, there are only a few life-course studies focusing on the effects of early childhood abuse on dental health outcomes in older age. With regard to dental health in middle age, Nicolau et al. (2007) reported that people with high paternal punishment in childhood had clinically examined poorer periodontal status at age $40^{12}$. Listl et al. (2014) reported that people with childhood financial hardship had poorer chewing ability at 50 years of age ${ }^{26}$. However, to our knowledge, no previous studies have examined the harmful effects of childhood abuse on dental health in older age. In particular, no study has examined the effect on tooth loss in middle or older age, which 
77 reflects one's dental health status across the life-course. We aimed to investigate the 78 association between experience of childhood abuse and the number of remaining teeth among older adults in Japan.

80

\section{$81 \quad$ Methods}

$82 \quad$ Study design and setting

83 This study was a retrospective cohort study investigating the association between 84 experience of childhood abuse and current dental health status in older age. We used 85 data from the Japan Gerontological Evaluation Study (JAGES) project, in particular 86 from the 2013 JAGES survey which was conducted between October 2013 and 87 February 2014. JAGES is an ongoing prospective cohort study aiming to investigate the 88 association between social and behavioral factors and community-dwelling older 89 people's health in multiple cities in $\mathrm{Japan}^{27}$. In the 2013 JAGES survey, 30 90 municipalities in 14 of the 47 prefectures in Japan were included. Self-administered 91 questionnaires were mailed to 193,694 community-dwelling residents aged $\geq 65$ years.

92 Random sampling from small administrative regions was used to select participants in 9317 large municipalities, while all eligible residents in 13 small municipalities were 94 included. One in five participants were randomly sent the questionnaire containing 95 questions on experience of childhood abuse $(\mathrm{N}=38,731)$.

97 Measurement

98 We used the number of remaining teeth for the outcome. The data were gathered using 99 the following single question: "How many remaining teeth do you have? Including teeth 100 covered by crowns. The total number of remaining teeth of an adult, including wisdom 
101 teeth, should be 32." Response choices were: $\geq 20$ teeth, 10-19 teeth, 5-9 teeth, 1-4 102 teeth, and no teeth. Self-reported number of teeth was used in another study ${ }^{28}$. We coded 103 these five categories from " $\geq 20$ teeth" as 1 to "No teeth" as 5 and used these as an 104 ordered variable for the analyses.

105 Our main predictor was experience of childhood abuse up to the age of 18 , 106 which was determined from the following three questions with response choices of 107 "Yes/No": "I was beaten by a parent and injured", (Answering "Yes" meant having an 108 experience of physical abuse), "I was insulted and verbally abused by a parent" 109 (Answering "Yes" meant having an experience of psychological abuse), and "I have 110 been loved by a parent" (Answering "No" meant having an experience of psychological 111 neglect). These questions were derived from the Adverse Childhood Experiences 112 Study $^{29}$. We defined having any one of these experiences as a main exposure.

113 The following variables were adjusted to estimate the independent effect of 114 childhood abuse: age (65-69, 70-74, 75-79, 80-84, and $\geq 85$ years old), sex 115 (male/female), economic adversity in childhood (Yes/No), years of education $(<6$, 6-9, 116 10-12, and $\geq 13$ ), longest occupation ("high occupational status": professional/technical 117 and administrative positions, and "low occupational status": clerical, sales/service and 118 skilled/labor positions, agriculture/forestry/fishery workers, the self-employed, other 119 occupations, and those with no occupation), and current household equivalent annual 120 income ("low": <16,000 USD, "middle": 16,000-24,999 USD, and "high": $\geq 25,000$ 121 USD; 100 JPY=1 USD), self-reported co-morbid status (diabetes/stroke/depression), 122 and smoking status (current smoker/cessation/never smoker). 
125 Multivariable ordered logistic regression models were fitted to estimate the 126 odds ratios (ORs) and 95\% confidence intervals (CIs) for having fewer remaining teeth.

127 We first examined crude association (model 1) and adjusted for age and sex (model 2).

128 Then, we constructed a model to evaluate how socioeconomic status (SES) explains the 129 association (model 3) and a model in which all covariates were adjusted (model 4). 130 Additionally, we examined the association between number of remaining teeth and each 131 type of childhood abuse experience as well as the total number of childhood abuse 132 experiences. People with missing information on the number of remaining teeth were 133 excluded from the analyses. Missing information on other variables was categorized as 134 dummy variables at first. Then, to account for bias due to missing information, we 135 assumed missing at random and created ten multiple imputed datasets using the 136 multivariate normal imputation method $^{30}$. All variables in our main models were 137 included in the imputation models. Estimated parameters were combined using Rubin's 138 combination methods ${ }^{31}$. All analyses were conducted using Stata 13.1 software (Stata 139 Corp, College Station, TX).

141 Ethical considerations

142 Ethical approval for this study was obtained from the Ethics Committee at Nihon 143 Fukushi University (13-14). The questionnaires with encrypted codes and explanations 144 of this study were sent to the participants by mail. Therefore, the researchers cannot 145 identify individual participants. The participants were informed that their participation 146 was voluntary and returning the self-administered questionnaire by mail was taken as 147 consent. 
150 Of the 38,731 people who were mailed the survey, 27,525 responded (response

151 rate $=71.1 \%$ ). After excluding those whose information on sex and age was missing or

152 invalid and those whose information on the number of remaining teeth was missing,

$15325,189(65.0 \%)$ participants were included in the final analyses (mean age was 73.9

154 (standard deviation $=6.2$, ranged between 65 and 100$)$; male $=46.5 \%)$. The prevalence

155 of any experience of childhood abuse was $14.8 \%$ (1.4\% physical abuse, $5.3 \%$

156 psychological abuse, $11.3 \%$ psychological neglect; $12.1 \%, 2.1 \%$, and $0.6 \%$ had one, two,

157 and three types of abuse, respectively). The characteristics associated with fewer

158 remaining teeth were having an experience of childhood abuse, older age, male sex,

159 having economic adversity in childhood, low years of education, low occupational

160 status, low income, diabetes, stroke, and current smoking (Table 1).

161 Ordered logistic regression analyses using multiply imputed datasets to account

162 for bias due to missing information showed that an experience of any type of childhood

163 abuse was significantly associated with fewer remaining teeth (model 1: OR [95\% CI] $=$

$1641.27[1.19,1.35])$ (Table 2). The association was significant after adjusting for age and

165 sex $($ model $2:$ OR $[95 \% \mathrm{CI}]=1.28[1.20,1.37])$. SES partly attenuated the association

166 (model 3: OR $[95 \% \mathrm{CI}]=1.15[1.08,1.23]$ ), and it remained significant after adjusting

167 for other covariates (model 4: OR $[95 \% \mathrm{CI}]=1.14[1.06,1.22]$ ). In addition, each type

168 of childhood abuse was significantly associated with fewer remaining teeth (Table 3).

169 The ORs increased as the number of different types of childhood abuse increased.

170 Analyses including missing predictor information as dummy variables showed similar

171 outcomes (Appendices $1 \& 2$ ).

172 
173

174

175

176

177

178

179

180

181

182

183

184

\section{Discussion}

We found a significant association between experience of childhood abuse and fewer remaining teeth in older age. Experience of childhood abuse increased the risk of having fewer remaining teeth by $14 \%$ after controlling for covariates. Each type of childhood abuse had a significant association, and a biological gradient was observed.

This is the first study to investigate the long-term adverse effect of childhood abuse on dental health in older age. We used large samples from 30 municipalities in Japan. However, this study has several limitations. First, recall bias may have been present since older respondents retrospectively answered questions about their experience of childhood abuse. However, we targeted community-dwelling, non-disabled older residents and Havari and Mazzonna (2011) reported that older people robustly remember their childhood circumstances ${ }^{32}$. In addition, we adjusted for depression, which can induce recall bias for experience of childhood abuse. Thus, we believe the problem of recall bias is not a serious flaw in this study. Second, we used a self-reported outcome. However, the validity of the self-reported number of remaining teeth was elucidated using clinical examination data from the sample of JAGES 2010 survey ${ }^{33}$. Third, we could not consider respondents' dental health status in childhood or their dental health behavior because of a lack of information. However, these are not confounders because it is unreasonable to assume that poor dental health or unhealthy dental health behavior causes child abuse. Rather, they are mediators that connect childhood abuse to poor dental health in older age. Thus, experience of childhood abuse is associated with fewer remaining teeth in older age even if the association is explained by poor dental health or unhealthy dental behavior. Fourth, because of lack of information, the reason and age of losing teeth were unknown. Furthermore specific 
clinical data on dental health status (e.g. periodontitis and dental caries) at each life stage would be useful to investigate the mechanisms whereby child abuse affects dental status in older age. Fifth, we could not evaluate sexual abuse because it might recall the severe traumatic experience. Sexual abuse is known as a severe risk factor for adult

201

202

203

204

Although there has been little focus on the association between experience of childhood abuse and dental health in older populations, our study is consistent with previous life-course studies on ACEs and dental health in adulthood ${ }^{26,34-36}$. Poulton et al. (2002) investigated the long-lasting negative influences of socioeconomic disadvantage on adult dental health; people who grew up in families with low SES had a threefold increase in adult periodontal disease and dental caries at the age of $26^{34}$. The association remained significant when Thomson et al. (2004) additionally considered dental health status in childhood ${ }^{35}$. However, these studies focused only on early adult or middle-aged populations. To our knowledge, our present study is the first to investigate the association between experience of childhood abuse and dental health in an older population.

One of the major reasons for tooth loss in Japan is dental caries and their sequela $^{37}$, and it is reasonable to expect that older people with experiences of childhood 
221 abuse to have more dental caries during childhood ${ }^{23,24,38}$. Children who are abused by 222 their caregivers tend to have dental neglect ${ }^{39}$. In addition, such children tend to prefer

223

224

225

226

227

228

229

230

231

232

233

234

235

236

237

238

239

240

241

242

243 comfort foods (such as these containing high sugar) and sweet taste because of their

high stress and damaged inhibitory control systems ${ }^{17,40}$. Unfortunately, these exposures,

which cause dental caries, have a major effect in this critical period because children's

young permanent teeth are more susceptible to demineralization ${ }^{41}$.

Another major reason for tooth loss in Japan is periodontal disease ${ }^{37}$, and child

abuse might influence fewer remaining teeth in older age via periodontal disease. A

study of a middle-aged population showed that adults who experienced high levels of

paternal punishment during childhood had poor periodontal status at the age of $40^{12}$.

Adults with childhood abuse experience are more likely to smoke ${ }^{13}$ and thus, easily

develop periodontal disease. In addition, some previous studies have suggested that

children with ACEs have impaired immune systems and altered hypothalamic function, which affects the adaptive responses of organs to stressors ${ }^{11}$. An epigenetic DNA modification is suggested to be associated with this change, which could last until adulthood $^{11}$. This could make people vulnerable to stress- and immune function-related diseases, such as periodontal disease ${ }^{12}$.

SES would be one of the other risk factors linking experience of childhood abuse and fewer remaining teeth in older age. Children who experience abuse are more likely to have low SES in adulthood and at older ages ${ }^{42}$. Low SES is associated with dental diseases and unhealthy behavior ${ }^{43}$. In fact, the association between experience of child abuse and number of remaining teeth in older age was partly explained by SES in the present study. 
Organization ${ }^{1}$. To reduce child abuse requires multi-strategy approaches operating at individual, social, societal and community levels ${ }^{2}$. Societal and community strategies include development of legal frameworks such as banning any physical punishment of programs for parents to improve their parenting skills. In Japan, home visitation programs at age of 1-2 months and age of 4 months are conducted, however, it is questionable whether two home visits would reduce parents' stress ${ }^{44}$. Individual strategies include increasing access to pre-and postnatal welfare services and support. Such multilevel strategies might be able to reduce child abuse.

\section{Conclusion}

256 This study showed that older people with experience of childhood abuse had also have a beneficial effect on oral health across the life-course.

262

263

\section{Acknowledgements}

264

265

This study used data from the Japan Gerontological Evaluation Study (JAGES), conducted by the Center for Well-being and Society, Nihon Fukushi University as one of their research projects. The authors greatly appreciate their work. We would also like to thank the participants in the survey. This study was supported by Health Labour 
268 Sciences Research Grants, Comprehensive Research on Aging and Health

269 (H26-Choju-Ippan-006, H25-Choju-Ippan-003, H25-Kenki-Wakate-015,

270 H25-Irryo-Shitei-003 (Fukkou), H24-Junkanki (Seishu)-Ippan-007,

271 H24-Choju-Wakate-009), from the Japanese Ministry of Health, Labour and Welfare, a

272 grant from the Department of Health and Human Services, Grants-in-Aid for Scientific

273 Research (20319338, 22390400, 23243070, 23590786, 23790710, 24390469, 24530698,

$27424653150,24683018,25253052,25870881,15 \mathrm{H} 01972,15 \mathrm{H} 04781$ ) from the Japan

275 Society for the Promotion of Science, a grant from the National Center for Geriatrics

276 and Gerontology (No. 24-17, No. 24-23, No. J09KF00804), the Research and

277 Development Grants for Longevity Science from AMED (Japan Agency for Medical

278 Research and development), and the Fixed Income Fund (AXA Life Insurance Co.,

279 Ltd.). The authors have no conflicts of interest. 


\section{References}

1. World Health Organization Regional Office for Europe. Review of social determinants and the health divide in the WHO European Region: final report. Copenhagen: World Health Organization Regional Office for Europe; 2013. 15-6 p.

2. World Health Organization. Preventing child maltreatment: a guide to taking action and generating evidence. Geneva: WHO Press; 2006. 1-6 p.

3. Fujiwara T, Kawakami N. Association of childhood adversities with the first onset of mental disorders in Japan: Results from the World Mental Health Japan, 2002-2004. J Psychiatr Res; 2011;45:481-7.

4. Kessler RC, McLaughlin KA, Green JG, Gruber MJ, Sampson NA, Zaslavsky AM, et al. Childhood adversities and adult psychopathology in the WHO world mental health surveys. Br J Psychiatry 2010;197:378-85.

5. Bellis MA, Hughes K, Leckenby N, Hardcastle KA, Perkins C, Lowey H. Measuring mortality and the burden of adult disease associated with adverse childhood experiences in England: a national survey. J Public Health $2015 ; 37: 445-54$.

6. Cable N. Life Course Approach in Social Epidemiology: An Overview, Application and Future Implications. J Epidemiol 2014;24:1-6.

7. Kuh D, Shlomo Y Ben. A Life Course Approach to Chronic Disease Epidemiology. Oxford: Oxford University Press; 2004. 
8. Nicolau B, Marcenes W. How will a life course framework be used to tackle wider social determinants of health? Community Dent Oral Epidemiol 2012;40:33-8.

9. da Fonseca MA, Feigal RJ, ten Bensel RW. Dental aspects of 1248 cases of child maltreatment on file at a major county hospital. Pediatr Dent 1992;14:152-7.

10. Naidoo S. A profile of the oro-facial injuries in child physical abuse at a children's hospital. Child Abuse Negl 2000;24:521-34.

11. Gonzalez A. The impact of childhood maltreatment on biological systems: Implications for clinical interventions. Paediatr Child Health 2013;18:415-8.

12. Nicolau B, Netuveli G, Kim JWM, Sheiham A, Marcenes W. A life-course approach to assess psychosocial factors and periodontal disease. J Clin Periodontol 2007;34:844-50.

13. Taha F, Galea S, Hien D, Goodwin RD. Childhood maltreatment and the persistence of smoking: a longitudinal study among adults in the US. Child Abuse Negl 2014;38:1995-2006.

14. Cowell RA, Cicchetti D, Rogosch FA, Toth SL. Childhood maltreatment and its effect on neurocognitive functioning: Timing and chronicity matter. Dev Psychopathol 2015;27:521-33.

15. Mason SM, Flint AJ, Field AE, Austin SB, Rich-Edwards JW. Abuse victimization in childhood or adolescence and risk of food addiction in adult women. Obesity 2013;21:E775-81.

16. Greenfield EA, Marks NF. Violence from parents in childhood and obesity in 
adulthood: Using food in response to stress as a mediator of risk. Soc Sci Med 2009;68:791-8.

17. Michels N, Sioen I, Braet C, Huybrechts I, Vanaelst B, Wolters M, et al. Relation between salivary cortisol as stress biomarker and dietary pattern in children. Psychoneuroendocrinology 2013;38:1512-20.

18. Costacurta M, DiRenzo L, Sicuro L, Gratteri S, De Lorenzo A, Docimo R. Dental caries and childhood obesity: analysis of food intakes, lifestyle. Eur J Paediatr Dent 2014;15:343-8.

19. Mikkilä V, Räsänen L, Raitakari OT, Pietinen P, Viikari J. Consistent dietary patterns identified from childhood to adulthood: the cardiovascular risk in Young Finns Study. Br J Nutr 2005;93:923-31.

20. Lien N, Lytle LA, Klepp KI. Stability in consumption of fruit, vegetables, and sugary foods in a cohort from age 14 to age 21. Prev Med 2001;33:217-26.

21. Matos-Gomes N, Katsurayama M, Makimoto FH, Santana LLO, Paredes-Garcia E, Becker MA d'Avila, et al. Psychological stress and its influence on salivary flow rate, total protein concentration and $\operatorname{IgA}, \operatorname{IgG}$ and $\operatorname{IgM}$ titers. Neuroimmunomodulation 2010;17:396-404.

22. Fejerskov O, Kidd E. Dental Caries: The Disease and Its Clinical Management. 2nd edn. Oxford: Blackwell Munksgaard; 2008. 189-208 p.

23. Valencia-Rojas N, Lawrence HP, Goodman D. Prevalence of Early Childhood Caries in a Population of Children with History of Maltreatment. J Public Health Dent 2008;68:94-101. 
24. Nicolau B, Marcenes W, Allison P, Sheiham A. The life course approach: Explaining the association between height and dental caries in Brazilian adolescents. Community Dent Oral Epidemiol 2005;33:93-8.

25. Nicolau B, Marcenes W, Sheiham A. The relationship between traumatic dental injuries and adolescents' development along the life course. Community Dent Oral Epidemiol 2003;31:306-13.

26. Listl S, Watt RG, Tsakos G. Early life conditions, adverse life events and chewing ability at middle and later adulthood. Am J Public Health 2014;104:5562.

27. Kondo K. Health Inequalities in Japan: An Empirical Study of Older People. Trans Pacific Press. Melbourne; 2010.

28. Matsuyama Y, Aida J, Takeuchi K, Tsakos G, Watt RG, Kondo K, et al. Inequalities of dental prosthesis use under universal healthcare insurance. Community Dent Oral Epidemiol 2014;42:122-8.

29. Felitti VJ, Anda RF, Nordenberg D, Williamson DF, Spitz AM, Edwards V, et al. Relationship of Childhood Abuse and Household Dysfunction to Many of the Leading Causes of Death in Adults. Am J Prev Med 1998;14:245-58.

30. Carpenter J, Kenward M. Multiple Imputation and its Application. 1st edn. Hoboken: John Wiley \& Sons; 2012. 75-164 p.

31. Rubin DB. Multiple Imputation for Nonresponse in Surveys. Hoboken: John Wiley \& Sons; 1987. 1-26 p.

32. Havari E, Mazzonna F. Can we trust older people's statement on their childhood 
circumstances? evidence from SHARELIFE. SHARE Working Paper Series $2011 ; 05-2011$.

33. Yamamoto T, Kondo K, Fuchida S, Aida J, Nakade M, Hirata Y. Validity of self-reported oral health variables : Aichi Gerontological Evaluation Study (AGES) project. Heal Sci Heal Care 2012;12:4-12.

34. Poulton R, Caspi A, Milne BJ, Thomson WM. Association between children's experience of socioeconomic disadvantage and adult health: a life-course study. Lancet 2002;360:1640-5.

35. Thomson W, Poulton R, Milne B, Capsi a, Broughton J, Ayers K. Socioeconomic inequalities in oral health in childhood and adulthood in a birth cohort. Community Dent Oral Epidemiol 2004;32:345-53.

36. Tsuboya T, Aida J, Kawachi I, Katase K, Osaka K. Early life-course socioeconomic position, adult work-related factors and oral health disparities: cross-sectional analysis of the J-SHINE study. BMJ Open 2014;4:e005701e005701.

37. Aida J, Ando Y, Akhter R, Aoyama H, Masui M, Morita M. Reasons for permanent tooth extractions in Japan. J Epidemiol 2006;16:214-9.

38. Nicolau B, Marcenes W, Bartley M, Sheiham A. A life course approach to assessing causes of dental caries experience: The relationship between biological, behavioural, socio-economic and psychological conditions and caries in adolescents. Caries Res 2003;37:319-26.

39. Montecchi PP, Di Trani M, Sarzi Amadè D, Bufacchi C, Montecchi F, Polimeni 
A. The dentist's role in recognizing childhood abuses: study on the dental health of children victims of abuse and witnesses to violence. Eur J Paediatr Dent $2009 ; 10: 185-7$.

40. Weafer J, Burkhardt A, de Wit H. Sweet taste liking is associated with impulsive behaviors in humans. Front Behav Neurosci 2014;8:228.

41. Lynch RJM. The primary and mixed dentition, post-eruptive enamel maturation and dental caries: a review. Int Dent J 2013;63 Suppl 2:3-13.

42. Zielinski DS. Child maltreatment and adult socioeconomic well-being. Child Abus Negl 2009;33:666-78.

43. Watt RG, Sheiham A. Integrating the common risk factor approach into a social determinants framework. Community Dent Oral Epidemiol 2012;40:289-96.

44. Fujiwara T, Natsume K, Okuyama M, Sato T, Kawachi I. Do home-visit programs for mothers with infants reduce parenting stress and increase social capital in Japan? J Epidemiol Community Health 2012;66:1167-76. 
Table 1. Participant demographic characteristics and number of remaining teeth before applying multiple imputations $(\mathrm{n}=25,189)$

\begin{tabular}{lrrrrrrr}
\hline & \multicolumn{5}{c}{ No. of remaining teeth } \\
\cline { 3 - 8 } & & Total & $20+$ & $10-19$ & $5-9$ & $1-4$ & 0 \\
& $\mathrm{n}$ & $\%$ & $\%$ & $\%$ & $\%$ & $\%$ \\
\hline Total & 25189 & 50.6 & 21.5 & 10.6 & 7.0 & 10.2 \\
Experience of childhood abuse & & & & & & \\
& No & 20211 & 52.3 & 21.3 & 10.3 & 6.6 & 9.5 \\
& Yes & 3730 & 46.6 & 22.0 & 11.4 & 8.2 & 11.8 \\
Missing & 1248 & 35.3 & 22.9 & 14.0 & 10.3 & 17.5 \\
& & & & & & & \\
& $65-69$ & 7139 & 63.9 & 20.4 & 7.4 & 4.1 & 4.2 \\
& $70-74$ & 7554 & 55.8 & 22.6 & 9.5 & 5.5 & 6.6 \\
& $75-79$ & 5585 & 44.4 & 22.3 & 12.7 & 8.6 & 12.1 \\
& $80-84$ & 3370 & 34.0 & 21.8 & 14.5 & 10.6 & 19.2 \\
& $\geq 85$ & 1541 & 21.8 & 18.7 & 14.8 & 14.8 & 29.9 \\
& & & & & & & \\
& Male years old) & 11700 & 49.0 & 21.4 & 10.8 & 7.5 & 11.3 \\
& Female & 13489 & 52.0 & 21.6 & 10.4 & 6.6 & 9.3
\end{tabular}

Economic adversity in

childhood

$\begin{array}{rrrrrrrr} & \text { No } & 12588 & 52.3 & 21.0 & 10.4 & 6.5 & 9.7 \\ & \text { Yes } & 11230 & 50.2 & 22.0 & 10.4 & 7.2 & 10.1 \\ \text { Years of education } & \text { Missing } & 1371 & 38.0 & 22.7 & 13.6 & 9.8 & 15.9 \\ & & & & & & & \\ & <6 & 418 & 23.9 & 19.6 & 14.8 & 12.4 & 29.2 \\ 10-9 & 9888 & 42.2 & 22.8 & 12.6 & 8.8 & 13.7 \\ \geq 13 & 9268 & 5131 & 61.4 & 19.4 & 8.1 & 5.2 & 5.9 \\ & \text { Missing } & 484 & 38.8 & 22.3 & 13.4 & 10.7 & 14.7\end{array}$

Occupation

$\begin{array}{rcccccc}\text { High occupational status } & 5346 & 57.3 & 20.6 & 8.9 & 5.8 & 7.5 \\ \text { Low occupational status } & 16731 & 50.5 & 21.3 & 10.7 & 7.0 & 10.5 \\ \text { Missing } & 3112 & 39.6 & 24.1 & 13.3 & 9.2 & 13.8\end{array}$

Income

$\begin{array}{rcccccc}\text { Low } & 7836 & 43.8 & 22.9 & 12.5 & 8.5 & 12.3 \\ \text { Middle } & 6347 & 56.3 & 21.2 & 8.7 & 6.0 & 7.8 \\ \text { High } & 6202 & 59.9 & 19.3 & 8.4 & 4.9 & 7.5\end{array}$

22 
$\begin{array}{lllllll}\text { Missing } & 4804 & 42.2 & 22.5 & 12.8 & 8.9 & 13.6\end{array}$

Diabetes

\begin{tabular}{rrrrrrrr} 
& No & 20395 & 51.3 & 21.4 & 10.4 & 7.0 & 9.9 \\
Yes & 3380 & 46.0 & 22.4 & 12.0 & 7.5 & 12.2 \\
Depression & Missing & 1414 & 51.7 & 21.1 & 11.0 & 6.2 & 10.0 \\
& & & & & & & \\
No & 23518 & 50.5 & 21.5 & 10.6 & 7.1 & 10.3 \\
Yes & 257 & 49.4 & 21.8 & 14.0 & 6.2 & 8.6 \\
Missing & 1414 & 51.7 & 21.1 & 11.0 & 6.2 & 10.0 \\
Stroke & & & & & & & \\
& No & 22948 & 50.8 & 21.6 & 10.5 & 7.0 & 10.1 \\
& Yes & 827 & 42.2 & 21.0 & 12.5 & 9.9 & 14.4 \\
& Missing & 1414 & 51.7 & 21.1 & 11.0 & 6.2 & 10.0 \\
& & & & & & & \\
& Current smoker & 2554 & 38.1 & 24.8 & 13.0 & 8.9 & 15.1 \\
& Cessation & 3895 & 49.1 & 22.1 & 11.5 & 7.2 & 10.2 \\
& Never smoker & 18350 & 53.0 & 20.8 & 10.0 & 6.7 & 9.5 \\
& Missing & 390 & 34.9 & 25.9 & 14.6 & 10.0 & 14.6 \\
\hline
\end{tabular}


1

2

3

4

5

6

7

8

9

10

11

12

13

14

15

16

17

18

19

20

21

22

23

24

25

26

27

28

29

30

31

32

33

34

35

36

37

38

39

40

41

42

43

44

45

46

47

48

49

50

51

52

53

54

55

56

57

58

59

60

Table 2. Odds ratios (ORs) and 95\% confidence intervals (CIs) for having fewer remaining teeth after applying multiple imputations $(\mathrm{n}=25,189)$

\begin{tabular}{cccccccccc}
\hline \multicolumn{2}{c}{ Model 1 } & \multicolumn{2}{c}{ Model 2 } & \multicolumn{2}{c}{ Model 3 } & \multicolumn{2}{c}{ Model 4 } \\
OR & $(95 \% \mathrm{CI})$ & OR & $(95 \% \mathrm{CI})$ & OR & $(95 \% \mathrm{CI})$ & OR & $(95 \% \mathrm{CI})$
\end{tabular}

Experience of childhood abuse

$\begin{array}{lccccccc}\text { No } 1.00 & \text { reference } & 1.00 & \text { reference } & 1.00 & \text { reference } & 1.00 & \text { reference } \\ \text { Yes } 1.27 & (1.19,1.35) & 1.28 & (1.20,1.37) & 1.15 & (1.08,1.23) & 1.14 & (1.06,1.22)\end{array}$

Model 1: crude OR

Model 2: model $1+$ age and sex were adjusted

Model 3: model $2+$ economic adversity in childhood, years of education, occupation, and income were adjusted

Model 4: model 3 + diabetes, depression, stroke, and smoking status were adjusted 
Table 3. Odds ratios (ORs) and 95\% confidence intervals (CIs) for having fewer remaining teeth, different type of childhood abuse and its accumulation; after applying multiple imputations ( $\mathrm{n}=25,189)$; each exposures were separately included into models

\begin{tabular}{ccccccccc}
\hline \multicolumn{2}{c}{ Model 1 } & \multicolumn{2}{c}{ Model 2 } & \multicolumn{2}{c}{ Model 3 } & & \multicolumn{2}{c}{ Model 4} \\
OR & $(95 \% \mathrm{CI})$ & OR & $(95 \% \mathrm{CI})$ & OR & $(95 \% \mathrm{CI})$ & OR & $(95 \% \mathrm{CI})$
\end{tabular}

Physical abuse

$\begin{array}{ccccccccc}\text { No } & 1.00 & \text { reference } & 1.00 & \text { reference } & 1.00 & \text { reference } & 1.00 & \text { reference } \\ \text { Yes } & 1.63 & (1.35,1.97) & 1.66 & (1.37,2.02) & 1.42 & (1.17,1.72) & 1.41 & (1.16,1.71)\end{array}$

Psychological abuse

$\begin{array}{ccccccccc}\text { No } & 1.00 & \text { reference } & 1.00 & \text { reference } & 1.00 & \text { reference } & 1.00 & \text { reference } \\ \text { Yes } & 1.23 & (1.11,1.36) & 1.30 & (1.18,1.44) & 1.21 & (1.09,1.35) & 1.20 & (1.08,1.34)\end{array}$

Psychological neglect

$\begin{array}{ccccccccc}\text { No } & 1.00 & \text { reference } & 1.00 & \text { reference } & 1.00 & \text { reference } & 1.00 & \text { reference } \\ \text { Yes } & 1.25 & (1.16,1.34) & 1.25 & (1.16,1.34) & 1.10 & (1.02,1.19) & 1.09 & (1.01,1.17)\end{array}$

Number of childhood abuse

$\begin{array}{cccccccccc}0 & 1.00 & \text { reference } & 1.00 & \text { reference } & 1.00 & \text { reference } & 1.00 & \text { reference } \\ 1 & 1.26 & (1.17,1.35) & 1.26 & (1.17,1.36) & 1.14 & (1.06,1.23) & 1.13 & (1.05,1.21) \\ \geq 2 & 1.32 & (1.14,1.52) & 1.38 & (1.20,1.60) & 1.20 & (1.04,1.40) & 1.19 & (1.03,1.38)\end{array}$

Model 1: crude OR

Model 2: model $1+$ age and sex were adjusted

Model 3: model $2+$ economic adversity in childhood, years of education, longest occupation, and income were adjusted

Model 4: model 3 + diabetes, depression, stroke, and smoking status were adjusted 
1

2

3

4

5

6

7

8

9

10

11

12

13

14

15

16

17

18

19

20

21

22

23

24

25

26

27

28

29

30

31

32

33

34

35

36

37

38

39

40

41

42

43

44

45

46

47

48

49

50

51

52

53

54

55

56

57

58

59

60

Appendix 1. Odds ratios (ORs) and 95\% confidence intervals (CIs) for having fewer remaining teeth with missing information on predictors included as dummy variables $(\mathrm{n}=25,189)$

\begin{tabular}{ccccccccc}
\hline \multicolumn{2}{c}{ Model 1 } & \multicolumn{2}{c}{ Model 2 } & \multicolumn{2}{c}{ Model 3 } & \multicolumn{2}{c}{ Model 4} \\
OR & $(95 \% \mathrm{CI})$ & OR & $(95 \% \mathrm{CI})$ & OR & $(95 \% \mathrm{CI})$ & OR & $(95 \% \mathrm{CI})$
\end{tabular}

Experience of childhood abuse

$\begin{array}{cccccccc}\text { No } 1.00 & \text { reference } & 1.00 & \text { reference } & 1.00 & \text { reference } & 1.00 & \text { reference } \\ \text { Yes } 1.27 & (1.19,1.35) & 1.29 & (1.20,1.37) & 1.15 & (1.07,1.23) & 1.13 & (1.06,1.21)\end{array}$

Model 1: crude OR

Model 2: model $1+$ age and sex were adjusted

Model 3: model $2+$ economic adversity in childhood, years of education, occupation, and income were adjusted

Model 4: model 3 + diabetes, depression, stroke, and smoking status were adjusted 
Appendix 2. Odds ratios (ORs) and 95\% confidence intervals (CIs) for having fewer remaining teeth, different type of childhood abuse and its accumulation; missing information on predictors was corded as dummy variables $(\mathrm{n}=25189)$; each exposures were separately included into models

\begin{tabular}{ccccccccc}
\hline \multicolumn{2}{c}{ Model 1 } & \multicolumn{2}{c}{ Model 2 } & \multicolumn{2}{c}{ Model 3 } & \multicolumn{2}{c}{ Model 4} \\
OR & $(95 \% \mathrm{CI})$ & OR & $(95 \% \mathrm{CI})$ & OR & $(95 \% \mathrm{CI})$ & OR & $(95 \% \mathrm{CI})$
\end{tabular}

Physical abuse

$\begin{array}{ccccccccc}\text { No } & 1.00 & \text { reference } & 1.00 & \text { reference } & 1.00 & \text { reference } & 1.00 & \text { reference } \\ \text { Yes } & 1.73 & (1.43,2.09) & 1.73 & (1.43,2.09) & 1.44 & (1.19,1.75) & 1.44 & (1.18,1.74)\end{array}$

Psychological abuse

$\begin{array}{ccccccccc}\text { No } & 1.00 & \text { reference } & 1.00 & \text { reference } & 1.00 & \text { reference } & 1.00 & \text { reference } \\ \text { Yes } & 1.25 & (1.13,1.39) & 1.32 & (1.19,1.47) & 1.23 & (1.11,1.37) & 1.22 & (1.10,1.36)\end{array}$

Psychological neglect

$\begin{array}{ccccccccc}\text { No } & 1.00 & \text { reference } & 1.00 & \text { reference } & 1.00 & \text { reference } & 1.00 & \text { reference } \\ \text { Yes } & 1.24 & (1.16,1.34) & 1.25 & (1.16,1.34) & 1.09 & (1.01,1.17) & 1.07 & (1.00,1.16)\end{array}$

Number of childhood abuse

$\begin{array}{cccccccccc}0 & 1.00 & \text { reference } & 1.00 & \text { reference } & 1.00 & \text { reference } & 1.00 & \text { reference } \\ 1 & 1.26 & (1.17,1.35) & 1.26 & (1.17,1.36) & 1.13 & (1.05,1.22) & 1.12 & (1.04,1.21) \\ \geq 2 & 1.32 & (1.15,1.52) & 1.40 & (1.21,1.61) & 1.21 & (1.05,1.40) & 1.19 & (1.03,1.38)\end{array}$

Model 1: crude OR

Model 2: model $1+$ age and sex were adjusted

Model 3: model $2+$ economic adversity in childhood, years of education, longest occupation, and income were adjusted

Model 4: model 3 + diabetes, depression, stroke, and smoking status were adjusted 


\section{Table legends}

Table 1. Participant demographic characteristics and number of remaining teeth before applying multiple imputations $(\mathrm{n}=25,189)$

Table 2. Odds ratios (ORs) and 95\% confidence intervals (CIs) for having fewer remaining teeth after applying multiple imputations $(\mathrm{n}=25,189)$

Model 1: crude OR

Model 2: model $1+$ age and sex were adjusted

Model 3: model 2 + economic adversity in childhood, years of education, occupation, and income were adjusted

Model 4: model 3 + diabetes, depression, stroke, and smoking status were adjusted

Table 3. Odds ratios (ORs) and 95\% confidence intervals (CIs) for having fewer remaining teeth, different type of childhood abuse and its accumulation; after applying multiple imputations ( $\mathrm{n}=25,189)$; each exposures were separately included into models

Model 1: crude OR

Model 2: model $1+$ age and sex were adjusted

Model 3: model $2+$ economic adversity in childhood, years of education, longest occupation, and income were adjusted

Model 4: model 3 + diabetes, depression, stroke, and smoking status were adjusted

Appendix 1. Odds ratios (ORs) and 95\% confidence intervals (CIs) for having fewer 
remaining teeth with missing information on predictors included as dummy variables (n $=25,189)$

Model 1: crude OR

Model 2: model $1+$ age and sex were adjusted

Model 3: model $2+$ economic adversity in childhood, years of education, occupation, and income were adjusted

Model 4: model 3 + diabetes, depression, stroke, and smoking status were adjusted

Appendix 2. Odds ratios (ORs) and 95\% confidence intervals (CIs) for having fewer remaining teeth, different type of childhood abuse and its accumulation; missing information on predictors was corded as dummy variables $(n=25189)$; each exposures were separately included into models

Model 1: crude OR

Model 2: model $1+$ age and sex were adjusted

Model 3: model $2+$ economic adversity in childhood, years of education, longest occupation, and income were adjusted

Model 4: model 3 + diabetes, depression, stroke, and smoking status were adjusted 